\title{
Thermorésistance des bactéries psychrotrophes du lait cru et de leurs protéinases
}

\author{
par \\ J. MOTTAR *
}

R és u m é

Deux souches de bactéries psychrotrophes gram-négatives, Pseudomonas P 104 et Flavobacterium P 108, capables de produire des protéinases exocellulaires extrêmement thermostables, ont été isolées de lait de mélange réfrigéré. Les bactéries mêmes sont très sensibles à la chaleur. Une destruction totale peut être obtenue par un traitement thermique modéré, par exemple de $30 \mathrm{~s}$ à $60^{\circ} \mathrm{C}$, de $5 \mathrm{~s}$ à $65^{\circ} \mathrm{C}$ ou de $1 \mathrm{~s}$ à $70^{\circ} \mathrm{C}$.

La thermorésistance des protéinases produites par les microorganismes isolés est suffisamment grande pour survivre pleinement à un traitement UHT. Les valeurs $\mathrm{D}$ à $140^{\circ} \mathrm{C}$ calculés étaient de respectivement 4,8 min pour la protéinase de Pseudomonas P 104 et 6,2 min pour celle de Flavobacterium P 108.

Mots clés:

Bactéries psychrotrophes - Protéinases - Thermorésistance.

\section{S u m m ary}

HEAT RESISTANT PROTEINASES

FROM BACTERIAL ORIGIN IN MILK

Two gram-negative, psychrotrophic strains of bacteria, Pseudodomonas $P 104$ and Flavobacterium $P$ 108, capable of producing extremely heat stable exocellular proteinases, were isolated from deepcooled milk. The bacteria involved are rather heat-sensitive. Complete destruction can be achieved by a mild heat treatment, such as $30 \mathrm{~s}$ at $60^{\circ} \mathrm{C}, 5 \mathrm{~s}$ at $65^{\circ} \mathrm{C}$ or $1 \mathrm{~s}$ at $70^{\circ} \mathrm{C}$.

\footnotetext{
* Station Laitière de l'Etat, Melle. Centre de Recherches Agronomiques de
} l'Etat, Gand (Belgique). 
The thermoresistance of the proteinases produced by the isolated micro-organisms is so high that a UHT treatment is fully or partly survived. A D-value at $140^{\circ} \mathrm{C}$ was calculated for both the Pseudomonas $P 104$ proteinase and the Flavobacterium P 108 enzyme; it amounted to $4.8 \mathrm{~min}$ and $6.2 \mathrm{~min}$ respectively.

Keys words :

Psychrotrophic bacteria - Proteinases - Heat resistance.

\section{INTRODUCTION}

La destruction des micro-organismes et la dénaturation de leurs enzymes ont une importance essentielle dans la fabrication du lait UHT. Des protéinases thermostables, en provenance de bactéries psychrotrophes, peuvent cependant être présentes en si grandes quantités dans le lait cru réfrigéré, que la conservabilité du lait UHT fabriqué à partir de ce lait cru peut s'en trouver considérablement réduite (Mottar et al., 1979).

Les recherches ayant rapport aux protéinases extracellulaires thermorésistantes se sont, jusqu'à nos jours, concentrées sur les souches de Pseudomonas (Mayerhofer et al, 1973; Kishonti, 1975; Adams, Barach et Speck, 1975; Alichanidis et Andrews, 1977; Richardson et Te Whaiti, 1978; Law, 1979; Marshall et Marstiller, 1981 ; Richardson, 1981 ; Leinmüller et Christophersen, 1982 ; Stepaniak et Fox, 1983). On ne connaît que peu concernant la stabilité à la chaleur d'autres bactéries gram-négatives psychrotrophes ainsi que de la thermorésistance de leurs protéinases extracellulaires.

Pour cette raison nous avons isolé, à partir de lait cru, une importante souche psychrotrophe, autre que Pseudomonas, et productrice de protéinases thermostables. Ensuite nous avons tâché de l'identifier et d'en déterminer la thermorésistance ainsi que la stabilité à la chaleur de ses enzymes protéolytiques. La thermostabilité a été décrite par des paramètres cinétiques et comparée avec les caractéristiques d'une souche de Pseudomonas.

Pour la détermination de l'activité protéolytique nous avons utilisé une nouvelle méthode, basée sur un substrat tripeptidique.

\section{MATERIEL ET METHODES}

\subsection{Isolation et identification de micro-organismes protéolytiques psychrotrophes}

Du lait cru a été étalé sur un milieu de caséinate (Speck, 1976) et incubé 10 jours à $7^{\circ} \mathrm{C}$. Les colonies nettement protéolytiques ont été sélectionnées et purifiées par des repiquages successifs, suivis 
d'incubation à $21^{\circ} \mathrm{C}$. Les cultures purifiées ont été conservées à $7^{\circ} \mathrm{C}$ sur Nutrient Agar incliné (NA).

Une classification des souches protéolytiques a été faite sur la base de: coloration de Gram, mobilité, test d'oxydase de Kovacs, conversion du glucose, formation de pigment, conversion du lactose, assimilation du citrate, réduction du nitrate, conversion de l'arginine, assimilation de l'amidon et hydrolyse de la gélatine (Buchanan et Gibbons, 1974 ; Mossel et Tamminga, 1980).

\subsection{Production, isolation et purification partielle de protéinases bactériennes thermostables}

Les bactéries psychrotrophes isolées ont été incubées $72 \mathrm{~h}$ à $21^{\circ} \mathrm{C}$ sur NA, puis transvasées 2 fois à l'aide de $1 \mathrm{ml}$ de solution physiologique stérile dans $100 \mathrm{ml}$ de Nutrient Broth. Ces cultures ont été incubées avec agitation pendant $72 \mathrm{~h}$ à $21^{\circ} \mathrm{C}$.

Après incubation, les cellules bactériennes ont été séparées par centrifugation à $10000 \mathrm{~g}$ pendant $15 \mathrm{~min}$ à $4^{\circ} \mathrm{C}$. Après filtration stérilisante sur membrane avec une porosité de 0,45 $\mu \mathrm{m}$, le liquide surnageant, contenant les protéinases exocellulaires, a été concentré 10 fois à l'aide de modules d'ultrafiltration Millipore CX-10 à limite nominale d'exclusion de 10000 daltons. Les solutions d'enzymes à conserver étaient stérilisées par filtration; ces solutions d'enzymes concentrées étaient ensuite conservées à $5^{\circ} \mathrm{C}$.

Un purification partielle des enzymes a été obtenue par précipitation au $\left(\mathrm{NH}_{4}\right)_{2} \mathrm{SO}_{4}$ (saturé à $50 \% ; 372 \mathrm{~g} / \mathrm{l}$ ), après quoi le mélange était gardé $12 \mathrm{~h}$ à $7^{\circ} \mathrm{C}$ (Marshall et Marstiller, 1981). Le précipité était séparé par centrifugation $(20000 \mathrm{~g})$ et redissous dans $20 \mathrm{ml}$ d'eau bidistillée stérile. Les sels étaient éliminés par dialyse pendant $24 \mathrm{~h}$ à $5^{\circ} \mathrm{C}$ en présence d'eau. L'échantillon était ensuite dialysé pendant $24 \mathrm{~h}$ dans un tampon phosphate de $0,1 \mathrm{M}, \mathrm{pH}$ 7,5. La solution d'enzyme, partiellement purifiée, était finalement filtrée sur un filtre à membrane de porosité $0,45 \mu \mathrm{m}$ et conservée à $5^{\circ} \mathrm{C}$.

\subsection{Chauffage de suspensions bactériennes}

Des cultures fraîches, obtenues sur milieu NA, des bactéries protéolytiques isolées ont été transvasées, à l'aide d'une solution physiologique stérile, dans du lait écrémé stérile On veillait à ce que le nombre de germes initial soit de $10^{6}-10^{7} / \mathrm{ml}$. Des portions de $1 \mathrm{ml}$ ont été rapidement portées à la température voulue dans un bain-marie bouillant, puis maintenues pendant un temps déterminé à cette température, également en bain-marie.

Les temps de chauffage requis pour atteindre des températures situées entre 50 et $60^{\circ} \mathrm{C}$ étaient inférieurs à $10 \mathrm{~s}$. Ils n'ont pas été pris en considération dans l'expression du temps de chauffage. Les températures étaient contrôlées à l'aide d'un thermomètre à thermocouple. Le refroidissement dans l'eau glacée avait lieu immédiatement après la période de chauffe. 


\subsection{Chauffage des solutions d'enzymes protéolytiques}

Pour déterminer si une protéinase faisait preuve d'un certain degré de résistance à la chaleur, $0,5 \mathrm{ml}$ de solution d'enzyme non purifiée était placée dans une éprouvette, puis immergée pendant 5 min dans un bain d'huile à $100^{\circ} \mathrm{C}$. L'activité enzymatique était déterminée avant et après le chauffage.

Pour l'étude de l'inactivation thermique des protéinases P 104 et P 108, des portions de $100 \mu \mathrm{l}$ de filtrat d'enzymes partiellement purifiées ont été introduites dans des éprouvettes (diamètre intérieur : $2 \mathrm{~mm}$; diamètre extérieur: $3 \mathrm{~mm}$ ), qui furent ensuite fermées à la flamme, puis chauffées, dans un bain d'huile, à des températures situées entre 100 et $150^{\circ} \mathrm{C}$ et, enfin, rapidement refroidies dans de l'eau glacée. Le temps de chauffage du liquide, mesuré par un thermocouple, était de 10 à $25 \mathrm{~s}$ et n'a pas fait l'objet d'une correction.

\subsection{Détermination de l'activité protéolytique}

L'activité protéasique a été déterminée par mesurage de la libération de p-nitroaniline (pNA) d'un substrat tripeptidique, composé de H-D-Ile-Pro-Arg.-pNA (Kabidiagnostica S-2288).

Dans ce but, $300 \mu l$ d'une solution d'enzyme est pipettée dans une cuvette thermostatisée. Le tout est chauffé à $37^{\circ} \mathrm{C}$. Ensuite, on ajoute $300 \mu \mathrm{l}$ d'une solution tampon $\mathrm{pH} 7,4$ (6,1 g tris(hydroxyméthyl) aminométhane et $0,7 \mathrm{~g} \mathrm{NaCl} / 1$ eau bidistillée), préchauffée à $37^{\circ} \mathrm{C}$. Après mélange on ajoute $300 \mu \mathrm{l}$ de la solution du substrat $(4,5 \mathrm{~m}$ mol/1 H-D-Ile-Pro-Arg.-pNA-2HCL) et on mesure immédiatement la densité optique à $405 \mathrm{~nm}$. Le témoin est constitué de $300 \mu \mathrm{l}$ d'eau, $300 \mu \mathrm{l}$ de solution tampon et $300 \mu \mathrm{l}$ de solution de substrat. L'évolution de la densité optique est suivie pendant $10 \mathrm{~min}$. L'activité protéasique est déduite de l'augmentation de l'adsorption (dA) par unité de temps (min) et est expérimentée

\section{RESULTATS ET DISCUSSION}

\subsection{Sélection des bactéries psychrotrophes productrices de pro- téinases}

Douze cultures fortement protéolytiques et dont le liquide surnageant manifestait encore nettement une activité protéolytique après 5 min de chauffage à $100^{\circ} \mathrm{C}$, ont été isolées du lait cru réfrigéré. Il est apparu que 6 d'entre elles appartenaient au genre Pseudomonas et 6 au genre Flavobacterium.

Nos résultats démontrent que, conjointement aux Pseudomonas spp., il existe d'autres bactéries psychrotrophes, comme Flavobacterium spp. capables de produire des protéinases extracellulaires thermostables. 
Ceci est confirmé par les observations de Griffiths, Phillips et Muir (1981), qui ont trouvé qu'une partie significative des psychrotrophes dans le lait cru est composée de bactéries autres que Pseudomonas spp. et qui sont tout aussi capables de produire des enzymes thermostables. Kishonti (1975) démontrait que 24 des 60 bactéries psychrotrophes, isolées à partir de lait cru, produisaient des protéinases extracellulaires conservant au moins $75 \%$ de leur activité après un traitement de chauffage de $30 \mathrm{~min}$ à $63^{\circ} \mathrm{C}$ et appartenant aux genres Pseudomonas, Alcaligenes et Achromobacter.

\subsection{Thermorésistance des cultures psychrotrophes de "Pseudo- monas 》 P 104 et « Flavobacterium » P 108}

Les protéinases de 2 souches: Pseudomonas P 104 et Flavobacterium $\mathrm{P} 108$ se sont révélées les plus résistantes à la chaleur. Ces cultures et leurs enzymes ont été retenues pour examen ultérieur.

L'inactivation des cultures psychrotrophes de Pseudomonas P 104 et Flavobacterium P 108 par la chaleur entre 50 et $60^{\circ} \mathrm{C}$ suit une réaction du premier ordre (fig. 1 a et 1 b).

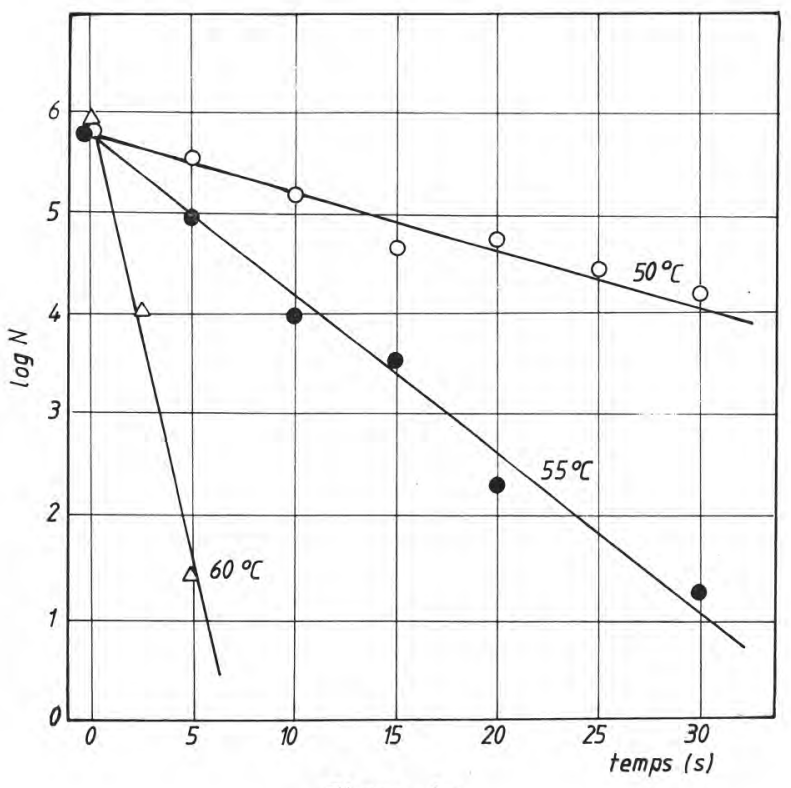

Figure 1 a

Inactivation thermique de la souche Pseudomonas P 104. 


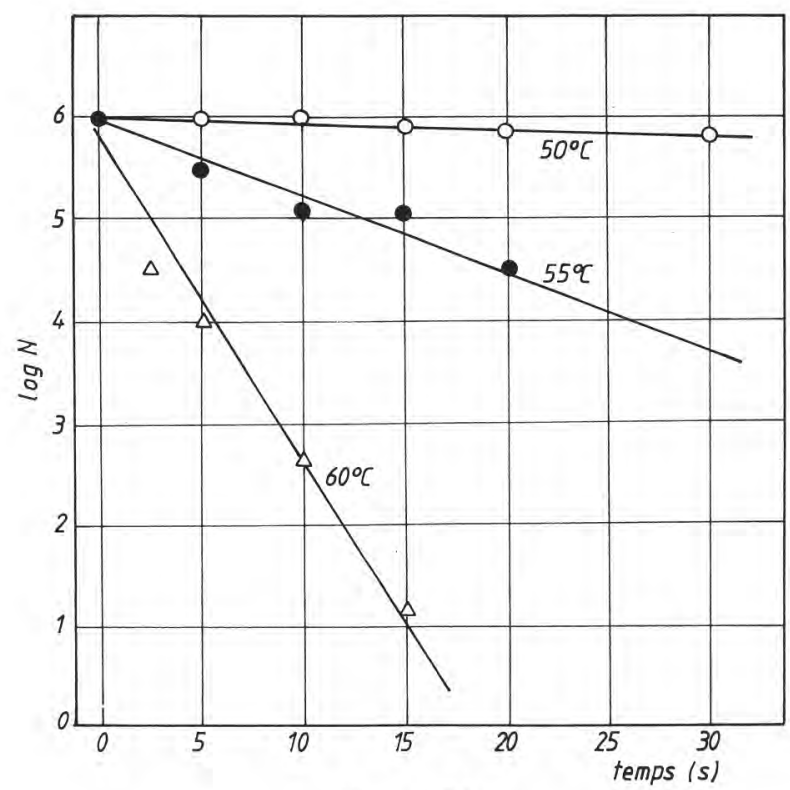

Figure $1 b$

Inactivation thermique de la souche Flavobacterium P 108.

Les données obtenues permettent de calculer la relation entre le temps de réduction décimale $\mathrm{D}$ et la température $\mathrm{T}\left({ }^{\circ} \mathrm{C}\right)$. Ces équations se présentent respectivement comme suit :

Pseudomonas $\mathrm{P} 104: \log \mathrm{D}=7,7142-0,1273 \mathrm{~T}(\mathrm{r}=-0,996)$;

Flavobacterium P $108: \log \mathrm{D}=10,6790-0,1706 \mathrm{~T}(=-0,983)$.

Partant de ces données, on peut calculer, pour l'intervalle de température de 50 à $75^{\circ} \mathrm{C}$, les temps de réduction décimale $\mathrm{D}$ et les constantes d'inactivation $\mathrm{k}$ (2,303/D), ainsi que la valeur de thermorésistance $\mathrm{z}$, c'est-à-dire la variation de température nécessaire pour obtenir l'augmentation au décuple ou la diminution au dixième de la valeur $D$, et le coefficient de température $Q_{10}\left(10^{10 / z}\right)$. Les paramètres cinétiques calculés pour les deux souches bactériennes en cause sont reproduits dans le tableau 1.

Des données bibliographiques donnent des valeurs $\mathrm{D}$ et $\mathrm{z}$ pour plusieurs Pseudomonas spp. (Kaufmann et Andrews, 1954 ; Chandary, Tuckey et Witter, 1960 ; Collins, 1961 ; Luedecke et Harmon, 1966). Cogan (1979) a calculé par extrapolation que les valeurs D à $71,1^{\circ} \mathrm{C}$, pour différents Pseudomonas spp., sont comprises entre 0,0001 et 0,324 s. Les valeurs $\mathrm{z}$ pour les Pseudomonas spp. se situent entre 3,7 et $7,9^{\circ} \mathrm{C}($ Cogan, 1979). 
TABLEAU 1 - TABLE 1

Paramètres cinétiques pour l'inactivation thermique des cellules entières de Pseudomonas P 104 et Flavobacterium P 108

\begin{tabular}{|c|c|c|c|c|c|}
\hline Souche bactérienne & $\mathrm{T}\left({ }^{\circ} \mathrm{C}\right)$ & $\mathrm{D}(\mathrm{s})$ & $\mathrm{k}\left(\mathrm{s}^{-1}\right)$ & $\mathrm{z}\left({ }^{\circ} \mathrm{C}\right)$ & $Q_{10}$ \\
\hline $\begin{array}{l}\text { Pseudomonas } \\
\text { P } 104\end{array}$ & $\begin{array}{l}50 \\
55 \\
60 \\
65 \\
70 \\
75\end{array}$ & $\begin{array}{r}21,26 \\
5,16 \\
1,19 \\
0,27 \\
0,063 \\
0,015\end{array}$ & $\begin{array}{c}0,108 \\
0,446 \\
1,931 \\
8,367 \\
36,23 \\
156,9\end{array}$ & 7,85 & 18,8 \\
\hline $\begin{array}{c}\text { Flavobacterium } \\
\text { P } 108\end{array}$ & $\begin{array}{l}50 \\
55 \\
60 \\
65 \\
70 \\
75\end{array}$ & $\begin{array}{c}140,92 \\
19,72 \\
2,77 \\
0,39 \\
0,05 \\
0,007\end{array}$ & $\begin{array}{c}0,016 \\
0,117 \\
0,830 \\
5,920 \\
42,20 \\
300,8\end{array}$ & 5,90 & 50,1 \\
\hline \multicolumn{6}{|c|}{$\begin{array}{l}\mathrm{D}=\text { Temps de réduction décimale } \\
\mathrm{k}=\text { Constante d'inactivation } \\
\mathrm{z}=\text { Valeur de thermorésistance } \\
\mathrm{Q}_{10}=\text { Coefficient de température }\end{array}$} \\
\hline
\end{tabular}

Les grandeurs cinétiques, trouvées pour Pseudomonas P 104 ainsi que pour Flavobacterium P 108, semblent être en concordance avec les données bibliographiques pour Pseudomonas spp.

La thermorésistance des micro-organismes gram-négatifs psychrotrophes se révèle très faible. Si l'on admet, comme il est généralement admis, qu'un processus de chauffage exerçant un effet destructeur équivalent à 10 valeurs $\mathrm{D}$ suffit pour exterminer totalement les bactéries présentes, on peut déduire des données acquises qu'une destruction totale des bactéries en cause est obtenue par chauffage de $30 \mathrm{~s}$ à $60^{\circ} \mathrm{C}$ ou de $5 \mathrm{~s}$ à $65^{\circ} \mathrm{C}$ ou de $1 \mathrm{~s}$ à $70^{\circ} \mathrm{C}$. Des chauffages de ce genre sont dès lors également indiqués pour le lait cru que l'on ne soumet pas au traitement UHT aussitôt après son arrivée à la laiterie.

\subsection{Thermorésistance des protéinases bactériennes exocellu- laires}

Dans l'intervalle de température $100-150^{\circ} \mathrm{C}$, l'inactivation thermique des protéinases exocellulaires de Pseudomonas P 104 et de Flavobacterium P 108, déterminées sur le surnageant de culture concentré et dialysé se produit selon une cinétique de réaction du premier ordre (fig. $2 \mathrm{a}$ et $2 \mathrm{~b}$ ). Elle montre que ces enzymes sont nettement thermostables. 


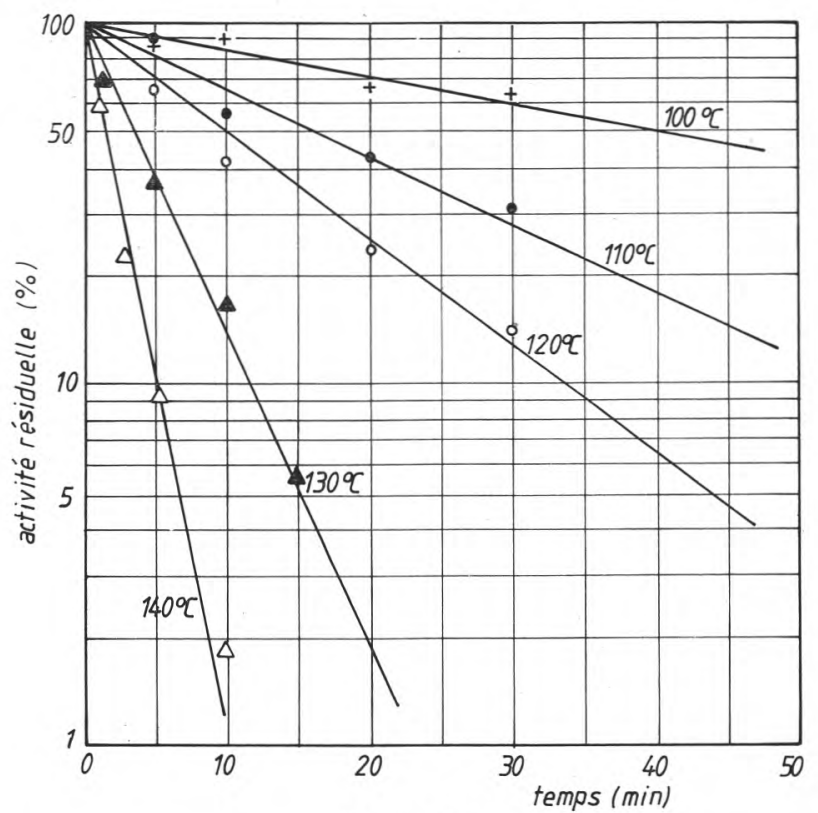

Figure $2 a$

Inactivation thermique de la protéinase P 104.

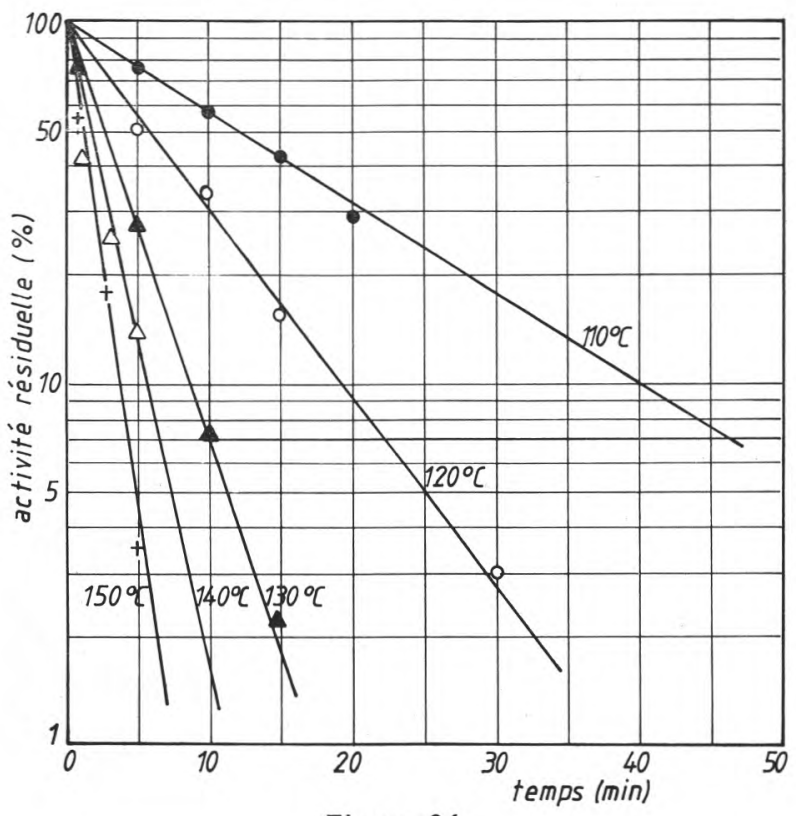

Figure $2 b$

Inactivation thermique de la protéinase P 108 . 
Pour les deux enzymes en question, la relation entre la température $\mathrm{T}\left({ }^{\circ} \mathrm{C}\right)$ et le temps de réduction décimale $\mathrm{D}$, pour l'intervalle de température susdit, peut être représentée par une droite :

- protéinase de P $104: \log \mathrm{D}=5,7380-0,0359 \mathrm{~T}(\mathrm{r}=-0,996)$,

- protéinase de P 108: $\log \mathrm{D}=4,5741-0,0273 \mathrm{~T}(\mathrm{r}=-0,992)$. De même que pour les cellules, des grandeurs cinétiques utiles ont été calculées (tableau 2).

TABLEAU 2 - TABLE 2

Paramètres cinétiques pour l'inactivation thermique des protéinases bactériennes P 104 et P 108

\begin{tabular}{|c|c|c|c|c|c|}
\hline $\begin{array}{l}\text { Protéi- } \\
\text { nase }\end{array}$ & $\mathrm{T}\left({ }^{\circ} \mathrm{C}\right)$ & $\mathrm{D}(\min )$ & $\mathrm{k}\left(\min ^{-1}\right)$ & $\mathrm{z}\left({ }^{\circ} \mathrm{C}\right)$ & $\mathrm{Q}_{10}$ \\
\hline P 104 & $\begin{array}{l}100 \\
110 \\
120 \\
130 \\
140\end{array}$ & $\begin{array}{r}139,6 \\
56,0 \\
33,2 \\
11,7 \\
4,8\end{array}$ & $\begin{array}{l}0,016 \\
0,041 \\
0,071 \\
0,197 \\
0,478\end{array}$ & 27,8 & 2,29 \\
\hline P 108 & $\begin{array}{l}110 \\
120 \\
130 \\
140 \\
150\end{array}$ & $\begin{array}{r}40,7 \\
18,9 \\
8,7 \\
6,4 \\
3,4\end{array}$ & $\begin{array}{l}0,056 \\
0,122 \\
0,266 \\
0,359 \\
0,768\end{array}$ & 36,6 & 1,87 \\
\hline
\end{tabular}

$\mathrm{D}=$ Temps réduction décimale

$\mathrm{k}=$ Constante d'inactivation

$\mathrm{z}=$ Valeur de thermorésistance

$\mathrm{Q}_{10}=$ Coefficient de température

La résistance à la chaleur des enzymes étudiées ici est telle que celles-ci peuvent résister pleinement ou en grande partie à un traitement UHT. Les valeurs z, publiées pour des souches de Pseudomonas, sont comprises entre 20 et 44,5 $\mathrm{C}$ (Mayerhofer et al., 1973 ; Adams, Barach et Speck, 1975; Kishonti, 1975; Driessen, 1976; Andrews et al., 1977 ; Cogan, 1979 ; Cerf, 1981 ; Richardson, 1981). Nos données concordent avec celles mentionnées dans ces références.

Il apparaît que l'inactivation des protéinases bactériennes thermorésistantes de $\mathrm{P} 104$ et $\mathrm{P} 108$ par la chaleur évolue, dans l'intervalle de température $100-150^{\circ} \mathrm{C}$, conformément à la théorie d'Arrhénius. 


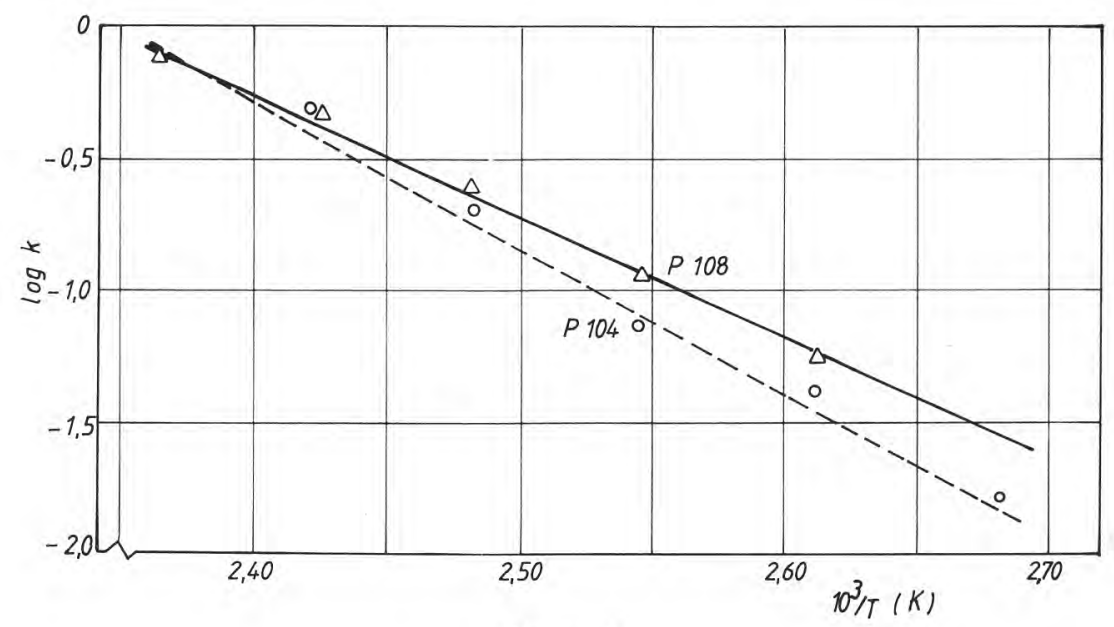

Figure 3

Inactivation thermique des protéinases P 104 et P 108 (Représentation d'Arrhénius). $[\mathrm{k}=$ pente des droites obtenues en exprimant log (activité résiduelle) en fonction du temps de chauffage (fig. 2 a et 2 b)]

En effet, comme il ressort de la figure 3, le rapport entre log $\mathrm{k}$ et $1 / \mathrm{T}$ est linéaire et peut être exprimé par une équation de la forme :

$$
\begin{aligned}
\mathrm{k} & =\mathrm{A} \cdot \mathrm{e}-\mathrm{E} / \mathrm{RT} \\
\text { où } \mathrm{k} & =\text { constante de vitesse }\left(\mathrm{min}^{-1}\right) \\
\mathrm{A} & =\text { constante } \\
\mathrm{E} & =\text { énergie d'activation }\left(\mathrm{J} \cdot \mathrm{mol}^{-1}\right) \\
\mathrm{R} & =\text { constante de gaz }\left(8,31 \mathrm{~J} \cdot \mathrm{mol}^{-1}\right) \\
\mathrm{T} & =\text { température absolue }(\mathrm{K}) .
\end{aligned}
$$

Les équations d'Arrhénius suivantes ont pu être calculées pour l'inactivation des protéinases examinées :

$$
\begin{aligned}
& \text { P 104: } \log \mathrm{k}=12,582-5,365 \cdot 10^{3} / \mathrm{T}(\mathrm{r}=0,996), \\
& \mathrm{P} 108: \log \mathrm{k}=10,398-4,451 \cdot 10^{3} / \mathrm{T}(\mathrm{r}=0,993) .
\end{aligned}
$$

De telles équations peuvent être utilisées pour prédire l'effet d'un traitement thermique sur l'activité des protéinases extracellulaires bactériennes dans le lait.

Il est important d'éviter la formation de protéinases thermorésistantes dans le lait, d'autant plus lorsque le lait est destiné à la production de produits UHT qui devront se conserver pendant une période relativement longue à température ambiante, car même un traitement UHT intensif ne peut pas inactiver totalement ces enzymes. 


\section{CONCLUSION}

Un traitement thermique modéré est suffisant pour éviter la production des protéinases thermorésistantes pendant le stockage du lait cru sous des conditions réfrigérées. Un procès de thermisation $\left(65^{\circ} \mathrm{C} / 10 \mathrm{~s}\right)$ empêche la croissance des bactéries psychrotrophes, ce qui est au point de vue énergétique plus favorable qu'une pasteurisation $\left(72-74^{\circ} \mathrm{C} / 15 \mathrm{~s}\right)$.

Les paramètres cinétiques d'inactivation laissent supposer que la destruction thermique des protéinases thermorésistantes exige des traitements thermiques qui nuiraient d'une manière inadmissible à la qualité du lait.

La méthode, basée sur un substrat tripeptidique, se révèle comme sensible et rapide pour une détermination précise de l'activité des solutions d'enzymes protéolytiques d'origine bactérienne.

\section{Bibliographie}

Adams (D. M.), Barach (J. T.) and SpeCK (M. L.) (1975). - Heat resistant proteases produced in milk by psychrotrophic bacteria of dairy origin. Journal of Dairy Science, 58, 828-634.

Alichanidis (E.) and ANDrews (A. T.) (1977). - Some properties of the extracellular protease produced by the psychrotrophic bacterium Pseudomonas fluorescens strain AR-11. Biochimica Biophysica Acta, 485, 424-430.

ANDrews (A. T.), LAW (B. A.) and Sharpe (M. E.) (1977). - An introduction to ultra-high temperature processing and plant. Journal of the Society of Dairy Technology, 30, 135-142.

Buchanan (R. E.) and Gibbons (N.E.) (1974). - Bergey's manual of determinative bacteriology, 8th ed. Williams and Wilkins Co., Baltimore.

Chandary (R. A.), Tuckey (S. L.) and Witter (L. D.) (1960). - Heat resistance of three strains of psychrophilic organisms added to skim-milk for cottage cheese manufacture. Journal of Dairy Science, 43, 1774-1779.

CERF (O.) (1981). - UHT treatment. International Dairy Federation, Document 133, $42-48$.

Cogan (T.M.) (1979). - Heat resistant lipases and proteinases and the quality of dairy products. International Dairy Federation, Document 43, 25-31.

Collins (E. B.) (1961). - Resistance of certain bacteria to cottage cheese cooking temperatures. Journal of Dairy Science, 44, 1989-1991.

DRIESSEN (F. M.) (1976), - Proteolysis in UHT sterilized milk and milk products. Zuivelzicht, 68, 514-515.

Griffitus (M. W.), Phillips (J.D.) and MuIR (D. D.) (1981). - Thermostability of proteases and lipases from a number of species of psychrotrophic bacteria of dairy origin. Journal of Applied Bacteriology, 50, 289-303.

KaufManN (O. W.) and ANDREws (R. H.) (1954). - The destruction rate of psychrophillic bacteria. Journal of Dairy Science, 37, 317-323.

KISHONTI (E.) (1975). - Influence of heat resistant lipases and proteases of psychrotrophic bacteria on product quality, International Dairy Federation, Document, $86,121-124$. 
Law (B. A.) (1979). - Review of the progress of Dairy Science: Enzymes of psychrotrophic bacteria and their effects on milk and milk products. Journal of Dairy Research, 46, 573-588.

Leinmuller (R.) and Christophersen (J.) (1982). - Beobachtungen zur Hitzeinaktiviering und Charakterisierung thermoresistenter Proteasen aus Pseudomonas fluorescens. Milchwissenschaft, 37, 472-475.

LUEDECKE (L. O.) and HARMON (L. G.) (1966). - Thermoresistance of Pseudomonas fragi in milk containing various amounts of fat. Applied Microbiology, 14, 716-720.

Marshall (R. T.) and Marstiller (J. T.) (1981). - Unique response to heat of extracellular protease of Pseudomonas fluorescens M5. Journal of Dairy Science, 64, 1545-1550.

MaYerhofer (H. J.), Marshall (R. T.), White (C. H.) and Lu (M.) (1973). Characterization of a heat stable protease of Pseudomonas fluorescens P 26. Applied Microbiology, 25, 44-48.

Mossel (D. A.) and Tamminga (S. K.) (1980). - Methoden voor het mikrobiologisch onderzoek van levensmiddelen. P.C. Noordervliet, Zeist.

Mottar (J.), Waes (G.), Moermans (R.) and Naudts (M.) (1979). - Sensoric changes in UHT milk during uncooled storage. Milchwissenschaft, 34, 257-262.

RichaRdSon (B. C.) (1981). - The purification and characterization of a heatstable protease from Pseudomonas fluorescens B 52. New Zealand Journal of Dairy Science and Technology, 16, 195-207.

Richardson (B. C.) and TE Whatti (T.E.) (1978). - Partial characterization of heat stable extracellular protease of some psychrotrophic bacteria from raw milk. New Zealand Journal of Dairy Science and Technology, 13, 172-176.

SPECK (M. L.) (1976). - Compendium of methods for microbial examination of Foods. American Public Health Association, Washington.

StePANIAK (L.) and Fox (P.) (1983). - The thermal stability of an extracellular proteinase form Pseudomonas fluorescence AFT 36. Journal of Dairy Research, 50, 171-184. 